giomata were seen in most and lung adenomata in all the mice; 2-aminofluorene caused thyomata. The closely related $N Z B$ strain did not react at all to urethane injection, and no papillomata were found. At the Australian centres in Adelaide and Sydney much the same type of cancer research is being done as in laboratories the world over, such as dosimetry, isotopes and calibration in radiation; carcinogens, chemotherapy, DNA, RNA, steroids in cancer; chromosomes in tumours and other tissues, tissue culture of malignant and normal cells, viruses, morphology and eytochemistry of neoplastic cells, environmental characteristics of cancer incidence.

Stoker in Glasgow has found that two types of response are elicited by polyoma virus acting on susceptible cells in vitro; in one shown by mouse embryo cells, the virus induces cell lysis with the production of large amounts of progeny virus, and in the second (a continuous line of hamster kidney fibroblasts) a small proportion of the cells are transformed by the virus infection and become malignant.

The report from the Glasgow Veterinary School is on lymphosarcoma in domestic animals-a virus has been grown in tissue culture from a lymph node of a feline lymphosarcoma.

I. HIEGER

\section{Radiobiology and Radiotherapeutics}

In the past two years radiobiology has made exceptional progress, and work supported by the Campaign has contributed in no small measure. The radiosensitivity of cells is influenced by many factors, both extrinsic and intrinsic; more of these are being identified and knowledge is being gained about some of the biochemical mechanisms responsible. Until recently, much of this work was done with cancer cells and these continue to provide valuable leads; exceptionally resistant cell lines have been isolated from growing culture (St. Bartholomew's Hospital) as well as from a spontaneous carcinoma (Mount Vernon); HeLa cells were shown to be capable of withstanding very large doses if cooled to $-196^{\circ} \mathrm{C}$. The determining factor in radiotherapeutic procedures is not, in general, the response of the tumour, but the dose which the normal tissue can tolerate. There is now an urgent noed to learn more about the response of normal cells and organs to irradiation.

At the London Hospital the functional state of the thyroid of the rat was shown to have an effect on its radiosensitivity. Growth, stimulated by a goitrogen, was halved by irradiation with $750 \mathrm{r}$. The $\mathrm{X}$-ray sensitivity was decreased if, at the time of irradiation, thyroid function was depressed by TSH. At Mount Vernon Hospital techniques have been developed to measure quantitatively the in vivo radiosensitivity of epithelial cells of mouse skin, which was found to fluctuate depending on previous exposure. Dividing cells in regenerating liver were about three times as resistant as those of skin. The sensitivity of mouse oocytes was shown to depend critically on the stage of maturation and there is an intermediate stage of high radioresistance (St. Bartholomew's Hospital). Paradoxical oxygen effects, such as protection by anoxia, have also been observed in the mouse ovary. Extensive investigations of the effects of $\mathrm{X}$-rays on growing bone are reported from Guy's Hospital and on cells in the developing retina from the Strangeways Laboratory. The response of the central nervous system is particularly interesting and seems to be much more marked in hypertensive than in normal rats (St. Mary's Hospital). Relatively small doses to the brain produce no detectable effects alone, but greatly reduce the capacity of subsequently induced surgical lesions to heal. Also at St. Mary's Hospital it was shown that cysteamine is capable of providing good protection to animals even in the most severe hypoxia.

Studies with micro-organisms most clearly indicate the role of the physiological state in determining radiosensitivity. The increase in sensitivity of spores after plating on agar was shown to be an effect of altered meta. bolism and not to uptake of water (Christie Hospital). The well known protective action of sulphydryl compounds suggested that there might be a correlation between the sulphydryl content of cells grown under different conditions and their radiation response; but none was found (Mount Vernon). The increase in radioresistance of cells of $E$. coli $B / r$ on passing from the logarithmic to the stationary phase of growth was shown to bo prevented by the addition of sulphanilamide (Chester Beatty Research Institute) because repair of a radiation lesion was prevented. Repair was also evoked as an explanation for the fact that the dose-response curve of Serratia marcescens to X-rays was not exponential with dose, but was a continuously curving function indicating that the probability of killing increases with dose (Mount Vernon).

Biochemical investigations at the Chester Beatty Research Institute led to the concept that a key factor in radiosensitivity is the capacity of the cell to restitute radiochemical damage sustained by the DNA. The complex enzymatic repair process is itself radiosensitive and no simple relationship between initial damage (that is, dose delivered) and cell death is therefore possible. Using the very radioresistant organism Micrococcus radiodurans, restoration of breaks in the main DNA chain induced by radiation were measured directly. The action of an exonuclease seems to be necessary before rejoining can occur and the enzyme involved may be isolated soon.

When fractionated doses are given, as in conventional radiotherapy, sub-lethal damage is important, that is, the first dose does not kill but makes the cells more susceptible to a second dose. It has been known for some years that mammalian cells can recover from this type of sub-lethal damage and the rate of recovery plays a part in determining the total effect of a fractionated course of radiation treatment. There are many other factors, however, including blood vessel damage, cell migration and vasodilation. Indeed, the sparing effect seen after fractionation due to sub-lethal recovery can be entirely reversed by physio-pathological factors (St. Bartholomew's Hospital). Because the first dose of radiation is likely to alter some of the many factors which influence radiosensitivity at the cellular level, radiobiology cannot at present substitute a scientific basis for the ad hoc approach of determining fractionation which is, as yet, all that is available to the radiotherapist. Work with experimental tumours, particularly primary tumours, however, has indicated variables that are likely to be particularly important. The response and adjustment to continuous irradiation of organs in vivo and of cell cultures in vitro is throwing much light on the complex interplay of factors involved in fractionation such as intracellular recovery, cell replacement, stem cell selection and changes in anatomical structure. Further progress in this difficult field is reported from the Biophysics Department of the Institute of Cancer Research. Related studies are being carried out at the Christie Hospital where, in addition to experimental projects, extra-corporeal irradiation of the blood of leukaemia patients is being tried.

It is impossible to do more than mention the very extensive body of work in the field of radiation chemistry. The new technique of pulse radiolysis pioneered at Mount Vernon and the Christie Hospital and now also taken up at St. Bartholomew's Hospital is providing detailed kinetics of the rate of reaction of many substances with the radicals produced by radiation in water. The nature and life-time of some intermediary radicals produced in such reactions have been identified and these studies have led to hypotheses of the mechanism of action of some well known radio sensitizing agents such as iodoacetamide. The nature and role of hydrogen atoms in these reactions have been difficult to disentangle from the reactions of other 
species produced in irradiated water, and at Cambridge this problem is being attacked in an ingenious manner using hydrogen atoms produced directly in a high frequency discharge. Both at Mount Vernon and the Christie Hospital, irradiation of tissue culture media has given rise to substances toxic to mammalian cells, but their nature is as yet quite unknown.

Biochemical lesions in irradiated cells or subcellular particles continue to be observed, but their significance remains obscure. The release of enzymes from mitochondria and lysosomes and the enzymatic formation of organic peroxides is reported from St. Bartholomew's Hospital. In lymphoma cells in vitro the rate of uridine incorporation into RNA is decreased within a few minutes of a dose as small as $200 \mathrm{r}$. This proved not to be a lesion involving RNA synthesis-which was quite normalbut an effect on the ribose nucleotide RNA precursor pool. A similar pool effect was also found for DNA precursors with lymphoma cells, but not with Hela cells (Chester Beatty Research Institute).

P. Alexander

\section{Clinico-pathology}

The clinical diagnosis, surgery, radiotherapy and chemotherapy of cancer have shown no noteworthy advances in the past year.

A number of interesting reports are presented under the heading of clinico-pathological studies including the (invariably) first class study from St. Mark's Hospital. From their work it appears that the incidence of a second (metachronous) carcinoma of the large intestine is too low to justify more extensive operations for the treatment of rectal and colonic cancer. Cases with polyps in the first specimen have a greater chance (approximately twice) of developing cancer in the residual bowel than cases in which such polyps are not found. Their results in early cancer of the rectum show the rarity with which it is diagnosed and show further that the disease is being diagnosed no earlier today than 35 years ago. In half the "early" rectal cancers, origin from a pre-existing benign tumour was shown histologically.

Several other clinical studies serve merely to confirm our general experience of the factors involved in the prognosis of cancer. The later the stage, the less differentiated the tumour, the more silent and inaccessible the tumour, and so on-the worse the outlook. Another study, involving the perfusion of operation specimens, confirms the vascular nature of tumours of the large bowel, and that perfused cytotoxic drugs would only reach the periphery - suroly a most elaborate demonstration of a commonplace of pathological and surgical knowledge. In some studies, however, new data are presented-information not available from reshuffling the old pack of cards, so to speak. The review of forty-six embryonal sarcomata by the Hospital for Sick Children, Great Ormond Street, reveals a better prognosis when the genito-urinary tract is the primary site, compared with the other major location, the head and neck. This type of behaviour is neither predictable nor explicable.

Lane-Brown and Melton, starting with the clinical observation that regression of malignant melanoma can occur in association with vaccinia infection, have made an electron microscope study of oncolysis in a malignant melanocyto-vaccinia system in vitro. They found that the virus enters the cell, and that the optimum temperature for cell destruction was $33^{\circ} \mathrm{C}$ rather than $37^{\circ} \mathrm{C}$. This probably explains the dermotropism shown by the virus.

The Bristol Bone Tumour Registry report is interesting, as are all well documented series of rare tumours. Price recounts the difficulties in the histological diagnosis of giant cell tumour of bone (osteoclastoma); although six of the forty-two behaved in a malignant manner, in only three were the original histological appearances sufficiently indicative of malignancy for the diagnosis to be made. (It is not so long ago that, in England, the osteoclastoma was classified as a benign tumour, adequately managed by curettage and packing the defect with bone chips.) In a further five cases a necessary amputation of the affected limb was carried out, although not for malignancy. A quarter of the patients in this series required amputation, and the tumour is clearly one that should command respect. Price recommends radical excision, particularly for giant cell tumours of major long bones.

At the cellular level of research Sylvia Lawlor's group present the results of chromosome studies in forty-three patients with polycythaemia vera. They found aneuploid cells in the bone marrow in untreated patients, but treat ment with phosphorus- 32 caused a partial reversion to euploidy in some cases. In the cases treated the bone marrow may be normal, show sporadic abnormalities of the type induced by radiation or show the evolution of stable clones of abnormal cells. Patients who developed a leukaemoid state may have a grossly abnormal chromosome complement.

Work on spontaneously occurring tumours in domestic animals is still lagging. The Glasgow Veterinary School has continued its study of lymphosarcoma in domestic animals. Their aims are to establish the national incidence of lymphosarcoma in each species, to determine whether "multiple case herds" exist here, as they do in Denmark (Bendixen), and to determine whether changes in blood leucocytes occur in each herd. So far no virus particles have been found in electron microscopy in field cases of lymphosarcoma in cats, dogs and cattle. They are continuing their transmission experiments in cats and tissue culture in the same animals.

Greenwood (Edinburgh Centre of Rural Economy) has completed his study of the possible effects of various contrasting environments on tumour incidence in domestic fowl; his results suggest that the differential response encountered may be due to genetic differences in susceptibility to adenocarcinoma existing in stocks of different origin.

Thompsett of the Royal Veterinary College and Allison of M.R.C., Mill Hill, are studing virus papillomatosis in a range of domesticated animals.

As soon as a new environmental carcinogon is proved to be a potent cause of malignant disease in man, no effort should be spared to eradicate the danger where practical. In the past few years the relationship of the absorption of asbestos to the subsequent development of malignant mesothelioma has become widely accepted and we may expect that workers in the social medicine field among others will now be active in clarifying the overall pattern of the disease. The Department of Therapeutics and Pharmacology of Queen's University of Belfast, investigating disease in the pipe-covering trade, in which asbestos is used, found that half the 105 deaths they studied were due to cancer; carcinoma of the bronchus was the most common, but mesothelioma of the pleura was unusually common.

Controversy on the status of the Burkitt lymphoma is still active. Briefly, this lymphosarcoma is frequent in certain geographical locations in Africa and most commonly presents with a dramatically large tumour of the cranial and facial skeleton. Children and adolescents are the victims, and despite regression with chemotherapeutic agents like vincristine, or irradiation, the disease pursues a fatal course. When the lymphosarcoma first came into prominence, it was thought to be a newly defined entity, and restricted to Africa; but a viral cause has not yet been demonstrated. The histological appearances are those seen in the lymphomata of childhood in white children in England and North and South America. It is interesting that this pattern is also frequent in canine or feline lymphoma; nevertheless the clinical presentation and the frequency of the disease in Africans are as yet unexplained.
A. I. LeVTene 